\title{
Cancer in Adolescents and Young Adults
}

\author{
Gregory H. Reaman, M.D. (Chairperson), * Janet Bonfiglio, R.N. (Rapporteur), $\dagger$ \\ Mark Krailo, Ph.D., $\ddagger$ Cameron K. Tebbi, M.D.,§Sanford Leikin, M.D., \\ Robert Ettinger, M.D., Tl Lonnie K. Zeltzer, M.D.,\# James B. Nachman, M.D.,** \\ Gaston K. Rivera, M.D.,†† Albert Aboulafia, M.D., 㧊 \\ and David S. Rosen, M.D., M.P.H.§§
}

Cancer is a significant health problem in the adolescent and young adult population. Confusion exists within the field of pediatric oncology with respect to the definition of childhood cancer, since most population-based incidence studies define childhood cancer on the basis of age alone. We recommend that the scope of pediatric oncology include the study and treatment of cancer in infants, children, adolescents, and young adults, recognizing that significant differences in the frequency of specific types of cancer exist within various age groups between 0 and 21 years of age.

We can expand our knowledge of the causes and treatment of adolescents and young adults with cancer (i.e, potentially unique etiologic and biologic issues, gains in survival, and the late sequelae of cancer therapy) only by the identification of such patients to a coordinated research base such as that offered by the pediatric cooperative groups and the entry of all potentially eligible patients on pediatric cooperative group clinical trials.

In an attempt to assess the success in the identification of adolescent and young adult patients 15 to 21 years of age, an analysis of data provided through the Los Angeles County Cancer Surveillance Program was undertaken.

Presented at the American Cancer Society Workshop on Children with Cancer, Naples, Florida, September 5-6, 1991.

From the *Children's National Medical Center, Washington, DC; the †American Cancer Society, Atlanta, Georgia; the fUniversity of Southern California, Los Angeles, California; the §St. Joseph's Cancer Institute, Tampa, Florida; the \|Childrens National Medical

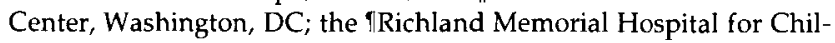
dren, Columbia, South Carolina; the \#University of California, Los Angeles, Los Angeles, California; the ${ }^{* *}$ University of Chicago, Chicago, Illinois; the $\dagger \dagger S t$. Jude Children's Research Hospital, Memphis, Tennessee; the $\ddagger$ Children's National Medical Center, Washington, DC; and the §̧̧University of Michigan, Ann Arbor, Michigan.

Address for reprints: Gregory H. Reaman, M.D., Children's National Medical Center, Department of Hematology-Oncology, 111 Michigan Avenue NW, Washington, DC 20010.

Accepted for publication March 26, 1992.
The analysis demonstrated that the percentage of patients identified to the pediatric cooperative groups decreased significantly as the age at the time of diagnosis increased. Whereas $60 \%$ of children age 0 to 9 years were registered with one of the two pediatric cooperative groups, only $17 \%$ of patients age 15 to 19 years were so identified. One explanation for this decline results from the observation that the diagnosis of cancer in older patients is more often made at institutions not affiliated with a cooperative group. This was not the sole explanation for this decrease, however. Indeed, when each of the six major cancer diagnostic categories (acute leukemia, brain tumors, lymphomas, soft tissue sarcomas, malignant germ cell tumors, and bone tumors) of children was considered, the registration rate of potentially eligible patients between the ages of 15 and 19 years was consistently lower than the registration rate of children between the ages of 0 and 14 years at institutions affiliated with cooperative groups.

More important, the same pattern was noted with respect to rates of enrollment on clinical trials in each of these disease categories. Concurrent analysis indicated that sex, race, and socioeconomic status were not related to these lower registration and enrollment rates. Furthermore, data from the SEER (Surveillance, Epidemiology and End Results), as well as the National Cancer Institute's (NCI) Cancer Therapy Evaluation Program, indicate that adolescents and young adults are infrequently registered with and entered on clinical trials of the adult cooperative groups. Since the resources available in a cooperative group setting represent significant advantages for the treatment of patients and the advancement of knowledge from basic and clinical research efforts in childhood and adolescent cancer, these striking differences in rates of registration and enrollment raise serious concerns about limited access to clinical trials for adolescents and young adult cancer patients between the ages of 15 and 21 years.

Investigation of potential explanations for these discrepant registration and enrollment rates should be 
immediately initiated. We further recommend that mechanisms be developed within cooperative group institutions for the timely identification of all cancer cases to clinical investigators at those institutions. Furthermore, methods of communication must be developed for smaller community-based institutions outside the cooperative group framework regarding the availability of appropriate pediatric cooperative group clinical trials. Such a system as Physician's Data Query (PDQ) should be used for this purpose and should be routinely linked to cancer diagnosis and subsequent treatment planning for adolescents and young adults. Furthermore, the Clinical Investigations Branch of the Division of Cancer Treatment, NCI must provide the leadership in developing policy and creating guidelines for the prioritization of patients on cooperative group clinical trials so that patients up to the age of 21 years would be routinely entered on trials of the pediatric cooperative groups in those situations where there are adult clinical trials that may compete for patient accrual. These guidelines should be disseminated through such groups as the American Society of Clinical Oncology, the American Cancer Society (ACS), and the American College of Surgeons Commission on Cancer. Data from the Children's Cancer Study Group and the St. Jude Children's Research Hospital demonstrate differences in outcome of adolescents and young adults when compared to younger children. Improved survival for this population with acute lymphoblastic leukemia (ALL), however, has been achieved in recent clinical trials and may be superior to survival outcome reported for patients aged 15 to 21 years on adult ALL trials. We recommend that comparative analysis of outcome data on current clinical trials of the pediatric and adult cooperative groups of young adults with acute leukemia, highgrade non-Hodgkin lymphoma, brain tumors, and soft tissue and bone sarcomas be conducted by the $\mathrm{NCI}$ and the ACS.

We recognize that approximately one-quarter of in. dividuals 15 to 21 years of age have tumors not routinely studied by pediatric cooperative groups (e.g., epithelial malignancies, carcinomas). We recommend that the entry of such patients on clinical trials sponsored by adult cooperative groups might be facilitated and that entry on these trials should not necessarily preclude the management of these patients at pediatric cancer centers where the special psychosocial and subspecialty expertise exists.

Since it is estimated that there will be more than 3500 cases of cancer diagnosed annually in adolescents aged 15 to 21 years, it is of paramount importance to identify these patients to ensure their optimal care and to protect an invaluable clinical research resource.

The management of adolescents and young adults with cancer poses significant and unique problems related to consent to therapy, delivery of prescribed therapy, and psychosocial concerns relevant to their successful integration into society and their transition to adulthood.

Legal and bioethical considerations governing clinical research do not recognize the autonomy of adolescents in decision making and require that adults give informed consent, which by convention is delegated to parents.

Research indicates that many adolescents after the age of 14 years have acquired the three principle criteria for making an informed decision: understanding, reasoning, and the ability to apply a set of values (conception of the good).

We recommend, therefore, that if the value set of an adolescent reflects his or her future interests, his or her decision-making competence should be assessed and, if deemed maximal, self-determination in decision making should be advocated. By empowering adolescents in decision making and in seeking their assent, chances for successful treatment are enhanced. Assent is defined as knowing agreement and requires full disclosure. The mere failure to object should not be construed as assent.

Major determinations in the evaluation of any therapy regimen include effective drugs, schedules, rates of administration, and compliance with prescribed therapy by patients as well as clinical investigators. The problem of compliance with self-administered therapy is particularly prevalent in adolescents and is felt to arise from the following three issues: confusion related to transfer of responsibility from parental control to self-determination; lack of parent-adolescent communication, and diminished parent-adolescent concordance.

There is confusion over the exact definition of compliance, although it has been defined as the "extent to which patient behavior coincides with medical or health advice." Allowing the adolescent to exercise self-determination with respect to assent to therapy should positively affect compliance. With respect to oral medication, compliance is best measured by querying the adolescent and young adult directly, rather than the parent. Recognizing that compliance is not easily measured objectively, we recommend that reproducible measures of evaluating and improving compliance be developed, studied through funded research, and applied. Since factors that affect compliance have been defined, we recommend that these be considered in the design and analysis of future clinical trials.

The following developmental tasks are required for the transition of the adolescent to adulthood: establishment of self-image, gaining independence, establishing 
peer relationships and sexual identity, and creating plans for the future. The impact of the diagnosis of cancer on the acquisition of these normal, age-appropriate developmental milestones and loss of control are responsible for the unique psychosocial concerns in this patient population. Since a major concern of adolescents diagnosed with cancer is that it interferes with their ability to communicate honestly and openly with their peers and parents, we recommend efforts be made to establish programs that facilitate their reintegration into peer groups, school, family, community, and workplace. Anecdotal experience suggests that interaction between adolescents successfully treated and newly diagnosed patients helps both groups deal with their disease more effectively. We recommend that psychosocial programs, specifically designed for adolescents and young adults, be developed within pediatric oncology programs. More important, funded research efforts must be initiated to define methods for their evaluation. Additional areas for research should include identification of the priority concerns of adolescent patients, risk factors for psychosocial maladjustment, and appropriate intervention strategies.

Not only are there short-term psychosocial concerns for adolescents and young adults with cancer, there are also long-term considerations in young adult survivors of childhood cancer. Although there does not appear to be an increase in diagnosable psychopathology, certain adjustment difficulties have been described that include exaggerated health care concerns, increased somatic complaints, excessive concerns related to development of secondary cancers, academic problems, delayed marriage, and excessive anxiety related to the health of future offspring. We recommend that funded research studies be developed to examine quality-of-life issues related to the successful accomplishment of life-style tasks, particularly during the pivotal period of transition from adolescence to adulthood.

As the prognosis for children, adolescents, and young adults with cancer improves, it is mandatory to consider how best to provide care to young adult cancer survivors. Since the global health care needs of adults are best met within the adult health care system, planning for transition from pediatric to adult-oriented health care systems is required. Strategies for planning and organizing the transition process must include early communication and education of patients and parents of the need for such transition, and requires that the provision of primary care occur outside of the subspecialty of pediatric oncology. Pediatric oncology programs, however, must facilitate access to primary adolescent and young adult health care by collaborative involvement with adolescent medicine specialists and internists.
Guidelines are available for the transition of young adults and adolescents with chronic disabilities through the Division of Maternal and Child Health. We recommend that these guidelines be evaluated in the development of specific transition plans for this population. Methods of evaluation of the efficacy of transition models have not been defined and require such definition. We recommend that funded research studies be undertaken to develop and evaluate models of transition at selected major pediatric cancer centers, including both free-standing children's hospitals and pediatric programs in large academic medical centers. We also recommend that transition planning be done to facilitate clinical research related to the evaluation of late effects of cancer therapy. Transition to an adult health care facility should be designed to facilitate and not compromise long-term follow-up studies in this patient population. In summary, it is recommended that:

1. The scope of the definition of pediatric oncology be expanded to include the study and treatment of cancer in infants, children, adolescents, and young adults.

2. Investigation of potential explanations for discrepant registration and enrollment rates of adolescents on clinical trials be initiated immediately.

3. Mechanisms be developed within cooperative group institutions for the timely identification of all cancer cases to clinical investigators at those institutions.

4. Methods of communication be developed for smaller community-based institutions outside the cooperative group framework regarding the availability of appropriate pediatric cooperative group clinical trials.

5. The Clinical Investigations Branch of the Division of Cancer Treatment, NCI provide the leadership in developing policy and creating guidelines for the age-related prioritization of patients on cooperative group clinical trials, and these guidelines be disseminated through such groups as the American Society of Clinical Oncology, the ACS, and the American College of Surgeons Commission on Cancer.

6. Comparative analysis of outcome data on current clinical trials of the pediatric and adult cooperative groups of young adults with acute leukemia, highgrade non-Hodgkin lymphoma, brain tumors, and soft tissue and bone sarcomas be conducted by the $\mathrm{NCI}$ and the ACS.

7. The entry of adolescent patients with tumors not routinely studied by pediatric oncologists (e.g., epithelial malignancies) on clinical trials sponsored by adult cooperative groups be facilitated and that entry on these trials not preclude the management 
of those patients at pediatric cancer centers where special psychosocial and subspecialty expertise exists.

8. Self-determination of adolescents in decision making regarding therapy and participation in clinical trials be advocated.

9. Reproducible measures of evaluating and improving compliance be developed, studied through funded research, and applied.

10. Efforts be made to establish programs that facilitate the reintegration of adolescents into peer groups, school, family, community, and workplace.

11. Psychosocial programs specifically designed for adolescents and young adults be developed within pediatric oncology programs.

12. Funded research efforts be initiated to define methods for evaluation of psychosocial programs designed for adolescents and young adults. Additional areas for research include identification of the priority concerns of adolescent patients, risk factors for psychosocial maladjustment, and appropriate intervention strategies.

13. Funded research studies be developed to examine quality-of-life issues related to the successful accomplishment of life-style tasks, particularly dur- ing the pivotal period of transition from adolescence to adulthood.

14. Pediatric oncology programs facilitate access to primary adolescent and young adult health care by collaborative involvement with adolescent medicine specialists and internists.

15. Guidelines developed by the Division of Maternal and Child Health be evaluated in the development of specific transition plans for this population.

16. Funded research studies be undertaken to develop and evaluate models of transition at selected major pediatric cancer centers, including both free-standing children's hospitals and pediatric programs in large academic medical centers.

17. Transition planning be done to facilitate clinical research related to the evaluation of late effects of cancer therapy.

18. The Workshop on Children and Cancer be renamed the Workshop on Cancer in Children, Adolescents, and Young Adults.

19. The American Cancer Society Task Force on Children and Cancer specifically address the issue of cancer in adolescents and young adults.

20. The American Cancer Society should conduct a Workshop on issues related to cancer in adolescents and young adults. 